\title{
HLA-A*2402-Restricted CDCA1-KIF20A Multipeptide Vaccine
}

National Cancer Institute

\section{Source}

National Cancer Institute. HLA-A*2402-Restricted CDCA1-KIF2OA Multipeptide Vaccine.

NCl Thesaurus. Code C90568.

A cancer vaccine containing two HLA-A $* 2402$-restricted peptide epitopes derived from cancer-testis antigens with potential immunostimulatory and antitumor activities. The peptide epitopes are derived from cell division associated 1 (CDCA1) and kinesin-like family member 20A (KIF20A). Upon administration, HLA-A*2402-restricted CDCA1KIF20A multipeptide vaccine may stimulate a cytotoxic T lymphocyte (CTL) response against CDCA1- and KIF20A-expressing tumor cells, resulting in tumor cell lysis and decreased tumor cell proliferation. HLA-A 2402 is an MHC class I molecule that presents antigenic peptides to CD8+ T cells; epitope design restricted to epitopes that bind most efficiently to HLA-A*2402 may improve antigenic peptide immunogenicity. 